\title{
molecules
}

ISSN 1420-3049

www.mdpi.com/journal/molecules

Article

\section{Total Synthesis of Six 3,4-Unsubstituted Coumarins}

\section{Wenqing Gao ${ }^{1}$, Qingyong $\mathrm{Li}^{1,2, *}$, Jian Chen ${ }^{1}$, Zhichao Wang ${ }^{1}$ and Changlong Hua ${ }^{1}$}

1 Key Laboratory of Forest Plant Ecology, Ministry of Education, Northeast Forestry University, Harbin 150040, China; E-Mails: gaowenqing1314@126.com (W.G.); 18042486759@189.com (J.C.); wzc870715@163.com (Z.W.); ghe521@163.com (C.H.)

2 College of Pharmaceutical Science, Zhejiang University of Technology, Hangzhou 310014, China

* Author to whom correspondence should be addressed; E-Mails: liqy@zjut.edu.cn or li_qingyong@126.com; Tel.: +86-571-8832-0984.

Received: 28 October 2013; in revised form: 8 December 2013 / Accepted: 10 December 2013 / Published: 13 December 2013

\begin{abstract}
In this article we describe a new methodology for the total synthesis of 3,4-unsubstituted coumarins from commercially available starting materials. Six examples were prepared, including five naturally occurring coumarins-7-hydroxy-6,8-dimethoxycoumarin (isofraxidin), 7-hydroxy-6-methoxycoumarin (scopoletin), 6,7,8-trimethoxycoumarin, 6,7-dimethoxycoumarin (scoparone), and 7,8-dihydroxycoumarin (daphnetin) and one synthetic coumarin, 7-hydroxy-6-ethoxycoumarin. Moreover, five important $o$-hydroxybenzaldehyde intermediates were also obtained, namely 2,4-dihydroxy-3,5dimethoxybenzaldehyde, 2,4-dihydroxy-5-methoxybenzaldehyde, 5-ethoxy-2,4-dihydroxybenzaldehyde, 2-hydroxy-3,4,5-trimethoxybenzaldehyde, and 2-hydroxy-4,5-dimethoxybenzaldehyde. The method developed herein involves just three or four steps and allows for the rapid synthesis of these important molecules in excellent yields. This is the first synthesis of 6,7,8-trimethoxycoumarin and 7-hydroxy-6-ethoxycoumarin.
\end{abstract}

Keywords: 3,4-unsubstituted coumarins; o-hydroxybenzaldehyde derivatives; 6,7,8-trimethoxycoumarin; 7-hydroxy-6-ethoxycoumarin

\section{Introduction}

Coumarins (2H-1-chromene-2-ones) are abundant in Nature and are common motifs found in drugs, dyes, spices, and agricultural chemicals. In particular, 3,4-unsubstituted coumarins are the most 
common naturally occurring coumarins, which show potential antimalarial [1], antioxidant [2-6], antimicrobial [7], anti-inflammatory [8], and antitumor [9-11] activity, however, their abundance in plants is very low and the purification processes are complex. Therefore, various methods have been developed for the total synthesis of coumarins [11-22], including the Perkin [23], Pechmann [24], and Knoevenagel reactions [25]. These reactions mostly lead to coumarins with substituents at the 3- or 4-position. A one-pot Wittig reaction/cyclization has been adopted by de Kimpe for the synthesis of 3,4-unsubstituted coumarins [26]. Herein, we report the highly efficient total synthesis of five naturally occurring coumarins, as well as a synthetic 3,4-unsubstituted coumarin (Figure 1). To the best of our knowledge, this is the first report on the total synthesis of coumarins 3 and $\mathbf{4}$.

Figure 1. The structure of the synthesized coumarins.<smiles>COc1cc2ccc(=O)oc2c(OC)c1O</smiles><smiles>COc1cc2ccc(=O)oc2cc1O</smiles>

Isofraxidin (1)<smiles>CCOc1cc2ccc(=O)oc2cc1O</smiles>

7-Hydroxy-6-ethyloxycouamrin (3)<smiles>COc1cc2ccc(=O)oc2c(OC)c1OC</smiles>

6,7,8-Trimethoxycoumarin (4)<smiles>COc1cc2ccc(=O)oc2cc1OC</smiles><smiles>O=c1ccc2ccc(O)c(O)c2o1</smiles>

Scoparone (5)

Daphnetin (6)

\section{Results and Discussion}

Coumarins 1, 2, and $\mathbf{3}$ were synthesized from simple starting materials (Scheme 1). The hydroxyl group was protected using pivaloyl chloride [27] to give the corresponding products in $100 \%$ yield. These compounds were then iodinated in $80 \%, 77 \%, 80 \%$ yields, respectively, using $\mathrm{N}$-iodo-succinimide [28,29], followed by hydrolysis using cuprous oxide, pyridine-2-aldoxime, tetrabutylammonium bromide, and cesium hydroxide [30]. The resulting $o$-hydroxybenzaldehydes were finally reacted with ethyl (triphenylphosphoranylidene) acetate in $N, N$-diethylaniline, forming coumarins $\mathbf{1}, \mathbf{2}$, and $\mathbf{3}$ as described above [26]. 
Scheme 1. Syntheses of coumarins 1, 2, and 3.<smiles>[R]c1cc(C=O)cc([R2])c1O</smiles><smiles>[R]c1cc(C=O)c(O)c([R])c1O</smiles>

Reagents and conditions: (a) $\left(\mathrm{CH}_{3}\right)_{3} \mathrm{COCl}$, DMAP, $\mathrm{Et}_{3} \mathrm{~N}, \mathrm{CH}_{2} \mathrm{Cl}_{2}$; (b) $10 \mathrm{NIS}, \mathrm{CF}_{3} \mathrm{COOH}, \mathrm{CH}_{3} \mathrm{CN}$, reflux; 11,12 $\mathrm{N}$-iodosuccinimide (NIS), $\mathrm{CF}_{3} \mathrm{SO}_{3} \mathrm{H}, \mathrm{MeOH}$; (c) $\mathrm{CsOH}$, syn-2-pyridinealdoxime, $\mathrm{Cu}_{2} \mathrm{O}, \mathrm{Bu}_{4} \mathrm{NBr}, \mathrm{N}_{2}$, $\mathrm{H}_{2} \mathrm{O}$; (d) $\left(\mathrm{C}_{6} \mathrm{H}_{5}\right)_{3} \mathrm{P}=\mathrm{CHCOOCH}_{2} \mathrm{CH}_{3}, \mathrm{Et}_{2} \mathrm{NPh}, \mathrm{N}_{2}$, reflux.

It was envisaged that this method could be applied to the synthesis of 6,7-dimethoxy-8hydroxycoumarin (34) and 5,6,7,8-tetrahydroxycoumarin (35) (Scheme 2).

Scheme 2. Attempted syntheses of coumarins 34, 35.<smiles>[R]c1c(C=O)c(I)c(OC)c(OC)c1C=O</smiles>

Reagents and conditions: (a) $26\left(\mathrm{CH}_{3}\right)_{3} \mathrm{COCl}$, DMAP, $\mathrm{Et}_{3} \mathrm{~N}, \mathrm{CH}_{2} \mathrm{Cl}_{2} ; 27 \mathrm{MeSO}_{4}, \mathrm{~K}_{2} \mathrm{CO}_{3}, \mathrm{CH}_{3} \mathrm{CN}$; (b) $28 \mathrm{NIS}$, $\mathrm{CF}_{3} \mathrm{COOH}, \mathrm{CH}_{3} \mathrm{CN}$, reflux; $29 \mathrm{NIS}, \mathrm{CF}_{3} \mathrm{SO}_{3} \mathrm{H}, \mathrm{MeOH}$; (c) $\mathrm{CsOH}$, syn-2-pyridinealdoxime, $\mathrm{Cu}_{2} \mathrm{O}, \mathrm{Bu}_{4} \mathrm{NBr}$, $\mathrm{N}_{2}, \mathrm{H}_{2} \mathrm{O}$. 
Protection of the hydroxyl groups using pivaloyl chloride and iodination of the resulting compounds proceeded as expected, but hydrolysis of the iodo-compounds $\mathbf{3 0}$ and $\mathbf{3 1}$ failed to afford the desired products 32 and 33. It was presumed that both electronic effects and steric hindrance of the three methoxy groups and the hydroxyl group at the ortho position of $\mathbf{3 0}$ and 31 affected the success of these reactions.

Synthesis of coumarins 4 and 5 (Scheme 3) began with the iodination of 3,4,5trimethoxybenzaldehyde (19) and veratraldehyde (20), respectively, both in 99\% yield. Hydrolysis of 2-iodo-3,4,5-trimethoxybenzaldehyde (21) and 2-iodo-4,5-dimethoxybenzaldehyde (22) afforded 2-hydroxy-3,4,5-trimethoxybenzaldehyde (23) and 2-hydroxy-4,5-dimethoxybenzaldehyde (24) in $83 \%$ and $85 \%$ yield, respectively. Finally, the $o$-hydroxybenzaldehydes $\mathbf{2 3}$ and $\mathbf{2 4}$ were converted to the corresponding coumarins $\mathbf{4}$ and $\mathbf{5}$. The overall yield of $\mathbf{4}$ and $\mathbf{5}$ was higher compared to that of compounds $\mathbf{1}-\mathbf{3}$.

Scheme 3. Syntheses of coumarins 4 and 5.<smiles>[R7]c1cc(C=O)cc(OC)c1OC</smiles>

Reagents and conditions: (a) $19 \mathrm{NIS}, \mathrm{CF}_{3} \mathrm{COOH}, \mathrm{CH}_{3} \mathrm{CN}$, reflux; 20 NIS, $\mathrm{CF}_{3} \mathrm{SO}_{3} \mathrm{H}, \mathrm{MeOH}$; (b) $\mathrm{CsOH}$, syn-2-pyridinealdoxime, $\mathrm{Cu}_{2} \mathrm{O}, \mathrm{Bu}_{4} \mathrm{NBr}, \mathrm{N}_{2}, \mathrm{H}_{2} \mathrm{O}$; (c) $\left(\mathrm{C}_{6} \mathrm{H}_{5}\right)_{3} \mathrm{P}=\mathrm{CHCOOCH}_{2} \mathrm{CH}_{3}, \mathrm{Et}_{2} \mathrm{NPh}, \mathrm{N}_{2}$, reflux.

Finally, daphnetin (6) was synthesized using the one-pot Wittig/cyclization reaction from commercially available 2,3,4-trihydroxybenzaldhyde in 65\% yield (Scheme 4).

Scheme 4. Synthetic procedures of coumarin 6.<smiles>CC(C)CC1CCCCC1</smiles>

Reagents and conditions: (a) $\left(\mathrm{C}_{6} \mathrm{H}_{5}\right)_{3} \mathrm{P}=\mathrm{CHCOOCH}_{2} \mathrm{CH}_{3}, \mathrm{Et}_{2} \mathrm{NPh}, \mathrm{N}_{2}$, reflux. 
This result for daphnetin (6) was identical compared to the previously reported synthesis [26]. Similarly for scopoletin (2), the overall yield of $50.4 \%$ (for four steps) is comparable to that of the previously reported synthesis (52.8\%) [24]. However, for isofraxidin (1) and scoparone (5), the yields of $57.6 \%$ and $77.4 \%$ are significantly higher compared to those obtained previously $[23,25]$. The key step in this novel method is the hydrolysis of the iodinated compound, as substituents on the phenyl group have a great influence on this reaction. Activating groups can reduce the probability of hydrolysis and steric hindrance at the ortho position also influences the reaction.

\section{Experimental}

All solvents and commercially available reagents were purchased from the suppliers and used without further purification. ${ }^{1} \mathrm{H}-\mathrm{NMR}$ and ${ }^{13} \mathrm{C}-\mathrm{NMR}$ spectra were recorded using Bruker DPX 500 (Bruker, Billerica, MA, USA) and 300 spectrometers, respectively. Spectra were recorded in $\mathrm{CDCl}_{3}$ and DMSO solutions and chemical shifts are reported in parts per million (ppm) relative to tetramethylsilane (TMS) as the standard. IR spectra were recorded on an Infinity Spectrum One spectrophotometer (Shimadzu, Kyoto, Japan). Mass spectra (Applied Biosystems, Toronto, Canada) were recorded on an Agilent 1100 Series VS (ES, 4000 V) mass spectrometer. Melting points were measured using a Büchi B-450 apparatus (Shanghaishenguang, Shanghai, China). Flash chromatography was performed with ACROS silica gel (particle size 0.030-0.040 mm, pore diameter ca. $6 \mathrm{~nm}$ ) using a glass column.

\subsection{General Procedure for the Synthesis of Coumarins 1-6}

The appropriate $o$-hydroxybenzaldehyde $(1 \mathrm{mmol})$ and ethyl(triphenylphosphoranylidene) acetate $(1.2 \mathrm{mmol})$ were dissolved in $N, N$-diethylaniline $(1.5 \mathrm{~mL})$ and the resulting mixture was stirred under a $\mathrm{N}_{2}$ atmosphere and reflux for $15 \mathrm{~min}$. The solvent was removed under reduced pressure $(1 \mathrm{mmHg}$, $52{ }^{\circ} \mathrm{C}$ ) and the resulting brown oil was purified by column chromatography (petroleum ether-ethyl acetate, $3: 1)$.

7-Hydroxy-6,8-dimethoxy-2H-chromen-2-one (Isofraxidin, 1). Yellow solid. Yield: 80\%. mp ( $\left.{ }^{\circ} \mathrm{C}\right)$ : 147-148.5 (lit. 148-149) [13]. ${ }^{1} \mathrm{H}-\mathrm{NMR}\left(500 \mathrm{MHz}, \mathrm{CDCl}_{3}\right): 3.95$ (s, 3H, $\left.\mathrm{OCH}_{3}\right), 4.10$ (s, 3H, $\left.\mathrm{OCH}_{3}\right)$, $6.16(\mathrm{~s}, 1 \mathrm{H}, \mathrm{Ar}), 6.29$ (d, $J=9.5 \mathrm{~Hz}, 1 \mathrm{H}, 3-\mathrm{CH}), 6.66(\mathrm{~s}, 1 \mathrm{H}, \mathrm{OH}), 7.60$ (d, $J=9.5 \mathrm{~Hz}, 1 \mathrm{H}, 4-\mathrm{CH})$; ${ }^{13} \mathrm{C}-\mathrm{NMR}\left(75 \mathrm{MHz}, \mathrm{CDCl}_{3}\right) 56.6\left(\mathrm{OCH}_{3}\right), 61.2\left(\mathrm{OCH}_{3}\right), 105.0(\mathrm{C}-5), 110.7(\mathrm{C}-4 \mathrm{a}), 112.5(\mathrm{C}-3)$, 135.2 (C-8), 143.5 (C-7), 144.5 (C-8a), 145.3 (C-4), 146.1 (C-6), 160.6 (C-2); IR (KBr, cm $\left.{ }^{-1}\right)$ $1699(\mathrm{C}=\mathrm{O}), 1570(\mathrm{C}=\mathrm{C}), 3327(\mathrm{br}, \mathrm{OH}) ; \operatorname{HRMS}(\mathrm{EI}): m / z 223\left[\mathrm{M}+\mathrm{H}^{+}\right]$.

7-Hydroxy-6-methoxy-2H-chromen-2-one (Scopoletin, 2). Yellow solid. Yield: 85\%. mp $\left({ }^{\circ} \mathrm{C}\right)$ : 202.7-204.4 (lit. 204) [15]. ${ }^{1} \mathrm{H}-\mathrm{NMR}\left(500 \mathrm{MHz}, \mathrm{CDCl}_{3}\right.$ ): 3.82 (s, 3H, $\mathrm{OCH}_{3}$ ), 6.23 (d, $J=9.5 \mathrm{~Hz}, 1 \mathrm{H}$, 3-CH), 6.79 (s, 1H, Ar), 7.23 (s, 1H, Ar), 7.92 (d, $J=9.5 \mathrm{~Hz}, 1 \mathrm{H}, 4-\mathrm{CH}), 10.33$ (s, 1H, OH); ${ }^{13} \mathrm{C}-\mathrm{NMR}$ $\left(75 \mathrm{MHz}, \mathrm{CDCl}_{3}\right) 56.4\left(\mathrm{OCH}_{3}\right), 103.2$ (C-8), 110.0 (C-5), 111.0 (C-3), 112.1 (C-4a), 144.9 (C-4), 145.7 (C-6), 150.0 (C-7), 151.6 (C-8a), $161.1(\mathrm{C}-2)$; IR $\left(\mathrm{KBr}, \mathrm{cm}^{-1}\right) 1558(\mathrm{C}=\mathrm{C}), 1683(\mathrm{C}=\mathrm{O})$, 3334 (br, OH). HRMS (EI): $m / z 193\left[\mathrm{M}+\mathrm{H}^{+}\right]$. 
7-Hydroxy-6-ethoxy-2H-chromen-2-one (3). Yellow solid. Yield: 80\%. mp $\left({ }^{\circ} \mathrm{C}\right)$ : 138.5-142.0. ${ }^{1} \mathrm{H}-\mathrm{NMR}\left(500 \mathrm{MHz}, \mathrm{CDCl}_{3}\right.$ ): 1.37 (t, $J=7.0 \mathrm{~Hz}, 3 \mathrm{H}, \mathrm{CH}_{3}$ ), 4.06 (q, $J=7.0 \mathrm{~Hz}, 2 \mathrm{H}, \mathrm{OCH}_{2^{-}}$), $6.23(\mathrm{~d}, J 9.5 \mathrm{~Hz}, 1 \mathrm{H}, 3-\mathrm{CH}), 6.81$ (s, 1H, Ar), 7.90 (d, J=9.5 Hz, 1H, 4-CH), 10.27 (s, 1H, CHO); ${ }^{13} \mathrm{C}-\mathrm{NMR}\left(75 \mathrm{MHz}, \mathrm{CDCl}_{3}\right) 15.1\left(\mathrm{CH}_{3}\right), 64.8\left(-\mathrm{CH}_{2} \mathrm{O}-\right), 103.2(\mathrm{C}-8), 111.0(\mathrm{C}-5), 111.2(\mathrm{C}-3)$, 112.1 (C-4a), 144.8 (C-4), 144.9 (C-6), 149.9 (C-7), 151.9 (C-8a), 161.1 (C-2); IR (KBr, cm $\left.{ }^{-1}\right)$ $1558(\mathrm{C}=\mathrm{C}), 1699(\mathrm{C}=\mathrm{O}), 1716,3383(\mathrm{br}, \mathrm{OH}) ; \mathrm{HRMS}(\mathrm{EI}): m / z 207\left[\mathrm{M}+\mathrm{H}^{+}\right]$.

6,7,8-Trimethoxy-2H-chromen-2-one (Dimethylfraxetin, 4). Yellow solid. Yield: 81\%. mp $\left({ }^{\circ} \mathrm{C}\right)$ : 103.8-104.5 (lit. 104-105) [31]. ${ }^{1} \mathrm{H}-\mathrm{NMR}\left(500 \mathrm{MHz}, \mathrm{CDCl}_{3}\right): 3.91$ (s, 3H, $\left.\mathrm{OCH}_{3}\right), 4.00$ (s, 3H, $\left.\mathrm{OCH}_{3}\right), 4.04\left(\mathrm{~s}, 3 \mathrm{H}, \mathrm{OCH}_{3}\right), 6.35(\mathrm{~d}, J=9.5 \mathrm{~Hz}, 1 \mathrm{H}, 3-\mathrm{CH}), 6.69(\mathrm{~s}, 1 \mathrm{H}, \mathrm{Ar}), 7.63(\mathrm{~d}, J=9.5 \mathrm{~Hz}, 1 \mathrm{H}$, 4-CH); ${ }^{13} \mathrm{C}-\mathrm{NMR}\left(75 \mathrm{MHz}, \mathrm{CDCl}_{3}\right) 56.3\left(\mathrm{OCH}_{3}\right), 61.5\left(\mathrm{OCH}_{3}\right), 61.8\left(\mathrm{OCH}_{3}\right), 103.7(\mathrm{C}-5)$, 114.3 (C-4a), 115.2 (C-8), 141.2 (C-6), 143.1 (C-7), 143.5 (C-4), 145.9 (C-8), 150.1 (C-8a), $160.5(\mathrm{C}-2)$; IR $\left(\mathrm{KBr}, \mathrm{cm}^{-1}\right) 1566(\mathrm{C}=\mathrm{C}), 1716(\mathrm{C}=\mathrm{O})$. HRMS (EI): $m / z 237\left[\mathrm{M}+\mathrm{H}^{+}\right]$.

6,7-Dimethoxy-2H-chromen-2-one (Scoparone, 5). Yellow solid. Yield: 91\%. mp $\left({ }^{\circ} \mathrm{C}\right)$ : 142.6-148.4 (lit. 145-146) [18]. ${ }^{1} \mathrm{H}-\mathrm{NMR}\left(500 \mathrm{MHz}, \mathrm{CDCl}_{3}\right)$ : $3.81\left(\mathrm{~s}, 3 \mathrm{H}, \mathrm{OCH}_{3}\right), 3.87\left(\mathrm{~s}, 3 \mathrm{H}, \mathrm{OCH}_{3}\right)$, $6.30(\mathrm{~d}, J=9.5 \mathrm{~Hz}, 1 \mathrm{H}, 3-\mathrm{CH}), 7.07$ (s, 1H, Ar), 7.25 (s, 1H, Ar), 7.955 (d, $J=9.5 \mathrm{~Hz}, 1 \mathrm{H}, 4-\mathrm{CH})$; ${ }^{13} \mathrm{C}-\mathrm{NMR}\left(75 \mathrm{MHz}, \mathrm{CDCl}_{3}\right) 56.3\left(\mathrm{OCH}_{3}\right), 56.6\left(\mathrm{OCH}_{3}\right), 100.5(\mathrm{C}-8) 109.3(\mathrm{C}-5), 111.6(\mathrm{C}-4 \mathrm{a})$, 113.1 (C-3), 144.8 (C-6), 146.3 (C-4), 149.9 (C-8a), 153.0 (C-7), 161.0 (C-2); IR (KBr, cm $\left.{ }^{-1}\right)$ $1516(\mathrm{C}=\mathrm{C}), 1558,1708(\mathrm{C}=\mathrm{O}), 1616,3566(\mathrm{br}, \mathrm{OH})$. HRMS (EI): $m / z 207\left[\mathrm{M}+\mathrm{H}^{+}\right]$.

7,8-Dihydroxy-2H-chromen-2-one (Daphnetin, 6). Yellow solid. Yield: 65\%. mp $\left({ }^{\circ} \mathrm{C}\right)$ : 265.7-267.2 (lit. 265-268) [21]. ${ }^{1} \mathrm{H}-\mathrm{NMR}\left(500 \mathrm{MHz}, \mathrm{CDCl}_{3}\right): 6.20(\mathrm{~d}, J=6.5 \mathrm{~Hz}, 1 \mathrm{H}, 3-\mathrm{CH}), 6.81$ (s, 1H, Ar), 7.03 (s, 1H, Ar), 7.91 (d, $J=7.0 \mathrm{~Hz}, 1 \mathrm{H}, 4-\mathrm{CH}), 9.79$ (d, $J=7.0 \mathrm{~Hz}, 1 \mathrm{H}, \mathrm{OH}), 9.80$ (d, $J=7.0 \mathrm{~Hz}, 1 \mathrm{H}$, $\mathrm{OH}) ;{ }^{13} \mathrm{C}-\mathrm{NMR}\left(75 \mathrm{MHz}, \mathrm{CDCl}_{3}\right) 111.7$ (C-3), 112.5 (C-4a), 112.9 (C-6), 119.3 (C-5), 132.6 (C-8), 144.2 (C-8a), 145.6 (C-4), 150.1 (C-7), 160.9 (C-2); IR (KBr, cm $\left.{ }^{-1}\right) 1674(\mathrm{C}=\mathrm{O}), 1508$ (C=C), 1589. HRMS (EI): $m / z 179\left[\mathrm{M}+\mathrm{H}^{+}\right]$.

\subsection{General Procedure for the Synthesis of Compounds 10-12}

The corresponding phenol (3 mmol) was dissolved in dichloromethane $(5 \mathrm{~mL})$ and 4-dimethylamiopyridine $(0.1 \mathrm{mmol})$ was added. The reaction was stirred for $0.5 \mathrm{~h}$ before addition of pivaloyl chloride $(6 \mathrm{mmol})$ followed by dropwise addition of triethylamine $(6 \mathrm{mmol})$. The reaction mixture was stirred at room temperature for $2 \mathrm{~h}$. The reaction solution was poured into dichloromethane $(100 \mathrm{~mL})$ and washed with saturated sodium chloride solution $(2 \times 100 \mathrm{~mL})$ and saturated sodium bicarbonate solution $(2 \times 100 \mathrm{~mL})$. The organic phase was collected, dried over anhydrous magnesium sulfate, and filtered, and then the solvent was removed in vacuo to obtain compounds 10-12.

4-Formyl-2,6-dimethoxyphenyl-2,2-dimethylpropanoate (10, $\left.\mathrm{C}_{14} \mathrm{H}_{18} \mathrm{O}_{5}\right)$. Yellow solid. Yield 100\%. $\mathrm{mp}\left({ }^{\circ} \mathrm{C}\right): 108-110.9 .{ }^{1} \mathrm{H}-\mathrm{NMR}\left(\mathrm{CDCl}_{3}, 500 \mathrm{MHz}\right): 1.39\left(\mathrm{~s}, 9 \mathrm{H}, \mathrm{C}\left(\mathrm{CH}_{3}\right)_{3}\right), 3.88\left(\mathrm{~s}, 6 \mathrm{H}, \mathrm{OCH}_{3}\right)$, $7.14(\mathrm{~s}, 2 \mathrm{H}, \mathrm{Ar}), 9.91(\mathrm{~s}, 1 \mathrm{H}, \mathrm{CHO}) ;{ }^{13} \mathrm{C}-\mathrm{NMR}\left(75 \mathrm{MHz}, \mathrm{CDCl}_{3}\right) 27.2\left(\mathrm{CH}_{3}\right), 29.7\left(\mathrm{CH}_{3}\right)$, 
$39.2(\mathrm{CC}=\mathrm{O}), 56.37\left(2 \times \mathrm{OCH}_{3}\right), 106.2(\mathrm{C}-3,5) 134.1(\mathrm{C}-1), 134.4(\mathrm{C}-4), 153.0(\mathrm{C}-2,6), 175.8(\mathrm{C}=\mathrm{O})$, 191.2 (CHO); IR (KBr, cm $\left.{ }^{-1}\right) 1751(\mathrm{C}=\mathrm{O}), 1691,1608$; MS (EI): $m / z 267\left[\mathrm{M}+\mathrm{H}^{+}\right]$.

4-Formyl-2-methoxyphenyl-2,2-dimethylpropanoate (11, $\left.\mathrm{C}_{13} \mathrm{H}_{16} \mathrm{O}_{4}\right)$. White solid. Yield: 100\%. mp $\left({ }^{\circ} \mathrm{C}\right)$ : 83.8-95.0. ${ }^{1} \mathrm{H}-\mathrm{NMR}\left(500 \mathrm{MHz}, \mathrm{CDCl}_{3}\right)$ : $1.38\left(\mathrm{~s}, 9 \mathrm{H}, \mathrm{C}\left(\mathrm{CH}_{3}\right)\right), 3.88\left(\mathrm{~s}, 3 \mathrm{H}, \mathrm{OCH}_{3}\right)$, 7.19 (d, $J=7.5 \mathrm{~Hz}, 1 \mathrm{H}, \mathrm{Ar}), 7.46$ (s, 1H, Ar), 7.48 (d, $J=7.5 \mathrm{~Hz}, 1 \mathrm{H}, \mathrm{Ar}), 9.95$ (s, 1H, CHO); ${ }^{13} \mathrm{C}-\mathrm{NMR}\left(75 \mathrm{MHz}, \mathrm{CDCl}_{3}\right) 27.2\left(3 \times \mathrm{CH}_{3}\right), 39.2(\mathrm{CC}=\mathrm{O}), 56.1\left(\mathrm{OCH}_{3}\right), 110.8(\mathrm{C}-2), 123.4(\mathrm{C}-6)$, 124.8 (C-5), 135.1 (C-1), 145.6 (C-4), 152.1 (C-3), 176.1 (-COO-), 191.2 (CHO); IR (KBr, cm $\left.{ }^{-1}\right)$ $1689(\mathrm{C}=\mathrm{O}), 1749$. MS (EI): $m / z 237\left[\mathrm{M}+\mathrm{H}^{+}\right]$.

2-Ethoxy-4-formylphenyl-2,2-dimethylpropanoate $\left(12, \mathrm{C}_{14} \mathrm{H}_{18} \mathrm{O}_{4}\right)$. White solid. Yield: $100 \%$. mp $\left({ }^{\circ} \mathrm{C}\right)$ : 83.8-85.3. ${ }^{1} \mathrm{H}-\mathrm{NMR}\left(500 \mathrm{MHz}, \mathrm{CDCl}_{3}\right)$ : $1.38\left(\mathrm{~s}, 9 \mathrm{H}, \mathrm{C}\left(\mathrm{CH}_{3}\right)_{3}\right), 1.41\left(\mathrm{~d}, J=7.0 \mathrm{~Hz}, 3 \mathrm{H}, \mathrm{CH}_{3}\right)$, $4.10\left(\mathrm{t}, J=7.0 \mathrm{~Hz}, 2 \mathrm{H}, \mathrm{OCH}_{2^{-}}\right), 7.19$ (d, $\left.J=8.5 \mathrm{~Hz}, 1 \mathrm{H}, \mathrm{Ar}\right), 7.46(\mathrm{~s}, 1 \mathrm{H}, \mathrm{Ar}), 7.46(\mathrm{~d}, J=7.0 \mathrm{~Hz}, 1 \mathrm{H}, \mathrm{Ar})$, $9.93(\mathrm{~s}, 1 \mathrm{H}, \mathrm{CHO}) ;{ }^{13} \mathrm{C}-\mathrm{NMR}\left(75 \mathrm{MHz}, \mathrm{CDCl}_{3}\right) 14.6\left(\mathrm{CH}_{3}\right), 27.2\left(\mathrm{CH}_{3}\right), 39.2(\mathrm{CC}=\mathrm{O}), 64.5\left(-\mathrm{OCH}_{2}-\right)$, 111.4 (C-2), 123.3 (C-6), 124.6 (C-5), 135.0 (C-1), 145.6 (C-4), 151.4 (C-3), $176.0(\mathrm{C}=\mathrm{O})$, $191.2(\mathrm{CHO}) ; \mathrm{IR}\left(\mathrm{KBr}, \mathrm{cm}^{-1}\right) 1699(\mathrm{C}=\mathrm{O}), 1602$; $\mathrm{MS}(\mathrm{EI}): \mathrm{m} / \mathrm{z} 251\left[\mathrm{M}+\mathrm{H}^{+}\right]$.

\subsection{General Procedure for the Synthesis of 13, 21, 22.}

The appropriate benzaldehyde $(3 \mathrm{mmol})$ was dissolved in acetonitrile $(5 \mathrm{~mL})$ and $N$-iodosuccinimide ( $6 \mathrm{mmol})$ was added. Trifluoroacetic acid $(0.3 \mu \mathrm{mol})$ was then added dropwise and the reaction mixture was heated under reflux for $6 \mathrm{~h}$. After cooling to room temperature, the reaction solution was quenched by the addition of sodium sulfite $(3 \mathrm{mmol})$ and stirred for five minutes. Subsequently, $\mathrm{CH}_{2} \mathrm{Cl}_{2}(100 \mathrm{~mL})$ was added and the solution was extracted with saturated sodium chloride solution $(3 \times 100 \mathrm{~mL})$. The organic phase was collected, dried over anhydrous magnesium sulfate, and filtered, and the solvent was removed in vacuo. Column chromatography (petroleum ether-ethyl acetate, 40:1) afforded the corresponding iodinated compound.

4-Formyl-3-iodo-2,6-dimethoxyphenyl-2,2-dimethylpropanoate $\left(\mathbf{1 3}, \mathrm{C}_{14} \mathrm{H}_{17} \mathrm{O}_{5}\right)$. Yellow solid. Yield: 80\%. $\mathrm{mp}\left({ }^{\circ} \mathrm{C}\right)$ : 100.7-102.5. ${ }^{1} \mathrm{H}-\mathrm{NMR}\left(500 \mathrm{MHz}, \mathrm{CDCl}_{3}\right): 1.40\left(\mathrm{~s}, 9 \mathrm{H}, \mathrm{C}\left(\mathrm{CH}_{3}\right)_{3}\right), 3.86\left(\mathrm{~s}, 3 \mathrm{H}, \mathrm{OCH}_{3}\right)$, $3.85\left(\mathrm{~s}, 3 \mathrm{H}, \mathrm{OCH}_{3}\right), 7.37$ (s, $\left.1 \mathrm{H}, \mathrm{Ar}\right), 10.08(\mathrm{~s}, 1 \mathrm{H}, \mathrm{CHO}) ;{ }^{13} \mathrm{C}-\mathrm{NMR}\left(75 \mathrm{MHz}, \mathrm{CDCl}_{3}\right) 27.1\left(\mathrm{CH}_{3}\right)$, $39.3(\mathrm{CC}=\mathrm{O}), 56.5\left(\mathrm{OCH}_{3}\right), 61.5\left(\mathrm{OCH}_{3}\right), 90.3(\mathrm{C}-2), 108.6(\mathrm{C}-6), 133.0(\mathrm{C}-4), 139.0(\mathrm{C}-1)$, 153.0 (C-5), $152.3(\mathrm{C}-3), 175.3(\mathrm{C}=\mathrm{O}), 191.0(\mathrm{C}=\mathrm{O}), 195.2(\mathrm{C}=\mathrm{O})$; IR $\left(\mathrm{KBr}, \mathrm{cm}^{-1}\right) 1691(\mathrm{C}=\mathrm{O}), 1759$; MS (EI): $m / z 393\left[\mathrm{M}+\mathrm{H}^{+}\right]$.

2-Iodo-3,4,5-trimethoxybenzaldehyde (21, $\left.\mathrm{C}_{10} \mathrm{H}_{11} \mathrm{O}_{3}\right)$. Yellow solid. Yield: 99\%. mp $\left({ }^{\circ} \mathrm{C}\right)$ : 67.6-68.1. ${ }^{1} \mathrm{H}-\mathrm{NMR}\left(500 \mathrm{MHz}, \mathrm{CDCl}_{3}\right): 3.97\left(\mathrm{~s}, 3 \mathrm{H}, \mathrm{OCH}_{3}\right), 3.92\left(\mathrm{~s}, 3 \mathrm{H}, \mathrm{OCH}_{3}\right), 3.91\left(\mathrm{~s}, 3 \mathrm{H}, \mathrm{OCH}_{3}\right)$, $7.35\left(\mathrm{~s}, 1 \mathrm{H}, \mathrm{OCH}_{3}\right), 10.05(\mathrm{~s}, 1 \mathrm{H}, \mathrm{CHO}) ;{ }^{13} \mathrm{C}-\mathrm{NMR}\left(75 \mathrm{MHz}, \mathrm{CDCl}_{3}\right) 61.2\left(\mathrm{OCH}_{3}\right), 61.0\left(\mathrm{OCH}_{3}\right)$, $56.3\left(\mathrm{OCH}_{3}\right), 91.6$ (C-2), 108.6 (C-6), 130.6 (C-1), 147.8 (C-4), 153.0 (C-5), 154.1 (C-3), $195.3(\mathrm{CHO}) ; \mathrm{IR}\left(\mathrm{KBr}, \mathrm{cm}^{-1}\right) 1687(\mathrm{C}=\mathrm{O})$; MS (EI): $m / z 323\left[\mathrm{M}+\mathrm{H}^{+}\right]$.

2-Iodo-4,5-dimethoxybenzaldehyde (22, $\left.\mathrm{C}_{9} \mathrm{H}_{9} \mathrm{O}_{2}\right)$. Yellow solid. Yield: 99\%. mp $\left({ }^{\circ} \mathrm{C}\right)::$ 145.1-145.5. ${ }^{1} \mathrm{H}-\mathrm{NMR}\left(500 \mathrm{MHz}, \mathrm{CDCl}_{3}\right): 3.92\left(\mathrm{~s}, 3 \mathrm{H}, \mathrm{OCH}_{3}\right), 3.96\left(\mathrm{~s}, 3 \mathrm{H}, \mathrm{OCH}_{3}\right), 7.31$ (s, 1H, Ar), 7.42 (s, 1H, Ar), 9.87 (s, 1H, Ar); ${ }^{13} \mathrm{C}-\mathrm{NMR}\left(75 \mathrm{MHz}, \mathrm{CDCl}_{3}\right) 56.1\left(\mathrm{OCH}_{3}\right), 56.5\left(\mathrm{OCH}_{3}\right), 92.8(\mathrm{C}-4), 111.1(\mathrm{C}-5)$, 
121.8 (C-1), 128.4 (C-6), 149.8 (C-3), 154.5 (C-2), 194.9 (CHO); IR (KBr, cm $\left.{ }^{-1}\right) 1672(\mathrm{C}=\mathrm{O})$; MS (EI): $m / z 293\left[\mathrm{M}+\mathrm{H}^{+}\right]$.

\subsection{General Procedure for the Synthesis of $\mathbf{1 4}$ and $\mathbf{1 5}$}

The appropriate benzaldehyde ( $3 \mathrm{mmol})$ was dissolved in methanol $(20 \mathrm{~mL})$ and $N$-iodosuccinimide (6 mmol) was added. Trifluoromethanesulfonic acid $(6 \mathrm{mmol})$ was then added dropwise and the reaction mixture was stirred at room temperature for $6 \mathrm{~h}$. The reaction was quenched by the addition of sodium sulfite and the reaction mixture was stirred for five minutes. Subsequently, dichloromethane (100 $\mathrm{mL})$ was added and the solution was extracted with saturated sodium chloride solution $(3 \times 100 \mathrm{~mL})$. The organic phase was collected, dried over anhydrous magnesium sulfate, and filtered; then, the solvent was removed in vacuo. Column chromatography (petroleum ether-ethyl acetate, 40:1) afforded the corresponding iodinated compound.

4-Formyl-5-iodo-2-methoxyphenyl-2,2-dimethylpropanoate (14, $\left.\mathrm{C}_{13} \mathrm{H}_{15} \mathrm{O}_{4}\right)$. Yellow solid. Yield: 77\%. $\mathrm{mp}\left({ }^{\circ} \mathrm{C}\right): 98.4 .{ }^{1} \mathrm{H}-\mathrm{NMR}\left(500 \mathrm{MHz}, \mathrm{CDCl}_{3}\right): 1.36\left(\mathrm{~s}, 9 \mathrm{H}, \mathrm{C}\left(\mathrm{CH}_{3}\right)_{3}\right), 3.86\left(\mathrm{~s}, 3 \mathrm{H}, \mathrm{OCH}_{3}\right), 7.50(\mathrm{~s}, 1 \mathrm{H}$, Ar), 7.58 (s, 1H, Ar), $9.96(\mathrm{~s}, 1 \mathrm{H}, \mathrm{CHO}) ;{ }^{13} \mathrm{C}-\mathrm{NMR}\left(75 \mathrm{MHz}, \mathrm{CDCl}_{3}\right) 27.1\left(3 \times \mathrm{CH}_{3}\right), 39.2(\mathrm{CC}=\mathrm{O})$, $56.2\left(\mathrm{OCH}_{3}\right), 89.6$ (C-2), 112.7 (C-6), 133.2 (C-3), 134.1 (C-1), 145.7 (C-4), 152.3 (C-5), 175.7 (-COO-), 194.9 (CHO); IR (KBr, cm $\left.{ }^{-1}\right) 1693$ (C=O), 1751; MS (EI): $m / z 363\left[{\left.\mathrm{M}+\mathrm{H}^{+}\right] .}^{\circ}\right.$

2-Ethoxy-4-formyl-5-iodophenyl-2,2-dimethylpropanoate (15, $\left.\mathrm{C}_{14} \mathrm{H}_{17} \mathrm{O}_{4}\right)$. Yellow solid. Yield: 80\%. mp $\left({ }^{\circ} \mathrm{C}\right): 81.7-83.8 .{ }^{1} \mathrm{H}-\mathrm{NMR}\left(500 \mathrm{MHz}, \mathrm{CDCl}_{3}\right): 1.37\left(\mathrm{~s}, 9 \mathrm{H}, \mathrm{C}\left(\mathrm{CH}_{3}\right)_{3}\right), 1.39(\mathrm{t}, J=7.0 \mathrm{~Hz}, 3 \mathrm{H}$, $\left.-\mathrm{CH}_{3}\right), 4.08$ (q, $\left.J=7.0 \mathrm{~Hz}, 2 \mathrm{H}, \mathrm{OCH}_{2}-\right), 7.47$ (s, 1H, Ar), 7.58 (s, 1H, Ar), 9.95 (s, 1H, CHO); ${ }^{13} \mathrm{C}-\mathrm{NMR}\left(75 \mathrm{MHz}, \mathrm{CDCl}_{3}\right) 14.5\left(\mathrm{CH}_{3}\right), 27.1\left(\mathrm{CH}_{3}\right), 39.2(\mathrm{CC}=\mathrm{O}), 64.7\left(-\mathrm{OCH}_{2}\right), 89.4(\mathrm{C}-2)$, 113.3 (C-6), 133.1 (C-3), 134.0 (C-1), 145.8 (C-4), 151.6 (C-5), 175.7 (C=O), 195.0 (CHO); IR $\left(\mathrm{KBr}, \mathrm{cm}^{-1}\right) 1685(\mathrm{C}=\mathrm{O}), 1759 ; \mathrm{MS}(\mathrm{EI}): \mathrm{m} / z 377\left[\mathrm{M}+\mathrm{H}^{+}\right]$.

\subsection{General Procedure for the Synthesis of 16-18, 23 and $\mathbf{2 4}$}

To the appropriate iodinated compound $(2 \mathrm{mmol})$ was added $\mathrm{Cu}_{2} \mathrm{O}(0.1 \mathrm{mmol})$, pyridine-2-aldoxime $(0.2 \mathrm{mmol})$, tetrabutylammonium bromide $(0.4 \mathrm{mmol})$, cesium hydroxide monohydrate $(10 \mathrm{mmol})$, and water $(2 \mathrm{~mL})$ and the mixture was stirred under $\mathrm{N}_{2}$ atmosphere for $10 \mathrm{~h}$. The reaction mixture was acidified to $\mathrm{pH}$ 5-7 with $1 \mathrm{M}$ hydrochloric acid. The aqueous layer was extracted with dichloromethane $(3 \times 100 \mathrm{~mL})$. The organic phases were combined, dried over anhydrous magnesium sulfate, and filtered; the solvent was then removed in vacuo. Column chromatography (petroleum ether-ethyl acetate: 20:1) afforded the corresponding phenol.

2,4-Dihydroxy-3,5-dimethoxybenzaldehyde (16, $\left.\mathrm{C}_{9} \mathrm{H}_{10} \mathrm{O}_{5}\right)$. Yellow solid. Yield: 90\%. $\mathrm{mp}\left({ }^{\circ} \mathrm{C}\right)$ : 87.5-89.8. ${ }^{1} \mathrm{H}-\mathrm{NMR}\left(500 \mathrm{MHz}, \mathrm{CDCl}_{3}\right): 3.91\left(\mathrm{~s}, 3 \mathrm{H}, \mathrm{OCH}_{3}\right), 4.01\left(\mathrm{~s}, 3 \mathrm{H}, \mathrm{OCH}_{3}\right), 6.41(\mathrm{~s}, 1 \mathrm{H}, \mathrm{Ar}), 6.75(\mathrm{~s}, 1 \mathrm{H}$, $\mathrm{OH}), 9.70(\mathrm{~s}, 1 \mathrm{H}, \mathrm{OH}), 11.30(\mathrm{~s}, 1 \mathrm{H}, \mathrm{CHO}) ;{ }^{13} \mathrm{C}-\mathrm{NMR}\left(75 \mathrm{MHz}, \mathrm{CDCl}_{3}\right) 56.6\left(\mathrm{OCH}_{3}\right), 60.9\left(\mathrm{OCH}_{3}\right)$, 108.9 (C-6), 112.9 (C-1), 134.6 (C-3), 141.2 (C-4), 146.9 (C-2), 151.6 (C-5), 194.6 (CHO); IR $\left(\mathrm{KBr}, \mathrm{cm}^{-1}\right) 3350$ (br, OH), $1650(\mathrm{C}=\mathrm{O})$; MS (EI): $\mathrm{m} / z 199\left[\mathrm{M}+\mathrm{H}^{+}\right]$. 
2,4-Dihydroxy-5-methoxybenzaldehyde $\left(\mathbf{1 7}, \mathrm{C}_{8} \mathrm{H}_{8} \mathrm{O}_{4}\right)$. Yellow solid. Yield: 77\%. mp $\left({ }^{\circ} \mathrm{C}\right): 152.1-153.2$. ${ }^{1} \mathrm{H}-\mathrm{NMR}\left(500 \mathrm{MHz}, \mathrm{CDCl}_{3}\right): 3.92\left(\mathrm{~s}, 3 \mathrm{H}, \mathrm{OCH}_{3}\right), 6.41$ (s, 1H, Ar), $6.53(\mathrm{~s}, 1 \mathrm{H}, \mathrm{Ar}), 6.89$ (s, 1H, OH), $9.68(\mathrm{~s}, 1 \mathrm{H}, \mathrm{OH}), 11.34(\mathrm{~s}, 1 \mathrm{H}, \mathrm{CHO}) ;{ }^{13} \mathrm{C}-\mathrm{NMR}\left(75 \mathrm{MHz}, \mathrm{CDCl}_{3}\right) 56.5\left(\mathrm{OCH}_{3}\right), 103.2(\mathrm{C}-3)$, 112.9 (C-1), 113.3 (C-6), 140.7 (C-5), 154.4 (C-4), 159.6 (C-2), 193.8 (CHO); IR (KBr, cm $\left.{ }^{-1}\right)$ 3292 (br, OH), $1635(\mathrm{C}=\mathrm{O})$; MS (EI): $m / z 169\left[\mathrm{M}+\mathrm{H}^{+}\right]$.

5-Ethoxy-2,4-dihydroxybenzaldehyde (18, $\left.\mathrm{C}_{9} \mathrm{H}_{10} \mathrm{O}_{4}\right)$. Yellow solid. Yield: $77 \%$. mp $\left({ }^{\circ} \mathrm{C}\right)$ : 131.8-134.4. ${ }^{1} \mathrm{H}-\mathrm{NMR}\left(500 \mathrm{MHz}, \mathrm{CDCl}_{3}\right.$ ): 1.47 (t, $J=7.0 \mathrm{~Hz}, 3 \mathrm{H}, \mathrm{CH}_{3}$ ), 4.12 (q, $J=7.0 \mathrm{~Hz}, 2 \mathrm{H}, \mathrm{OCH}_{2}$ ), $6.49(\mathrm{~s}, 1 \mathrm{H}, \mathrm{Ar}), 6.53(\mathrm{~s}, 1 \mathrm{H}, \mathrm{Ar}), 6.88(\mathrm{~s}, 1 \mathrm{H}, \mathrm{OH}), 9.65(\mathrm{~s}, 1 \mathrm{H}, \mathrm{OH}), 11.33(\mathrm{~s}, 1 \mathrm{H}, \mathrm{CHO}) ;{ }^{13} \mathrm{C}-\mathrm{NMR}$ $\left(75 \mathrm{MHz}, \mathrm{CDCl}_{3}\right) 14.8\left(\mathrm{CH}_{3}\right), 65.3\left(-\mathrm{CH}_{2} \mathrm{O}\right), 103.1$ (C-3), 113.3 (C-1), 113.9 (C-6), 139.9 (C-5), 154.6 (C-4), 159.5 (C-2), 193.8 (CHO); IR (KBr, cm $\left.{ }^{-1}\right) 1627$ (C=O), 1699, 3367 (br, OH); MS (EI): $m / z 183\left[\mathrm{M}+\mathrm{H}^{+}\right]$.

2-Hydroxy-3,4,5-trimethoxybenzaldehyde (23, $\left.\mathrm{C}_{10} \mathrm{H}_{12} \mathrm{O}_{4}\right)$. Yellow solid. Yield: 83\%. mp $\left({ }^{\circ} \mathrm{C}\right)$ : 46.0-46.6. ${ }^{1} \mathrm{H}-\mathrm{NMR}\left(500 \mathrm{MHz}, \mathrm{CDCl}_{3}\right): 3.87\left(\mathrm{~s}, 3 \mathrm{H}, \mathrm{OCH}_{3}\right), 3.94\left(\mathrm{~s}, 3 \mathrm{H}, \mathrm{OCH}_{3}\right), 4.05\left(\mathrm{~s}, 3 \mathrm{H}, \mathrm{OCH}_{3}\right)$, $6.78(\mathrm{~s}, 1 \mathrm{H}), 9.78(\mathrm{~s}, 1 \mathrm{H}, \mathrm{OH}), 11.01(\mathrm{~s}, 1 \mathrm{H}, \mathrm{CHO}) ;{ }^{13} \mathrm{C}-\mathrm{NMR}\left(75 \mathrm{MHz}, \mathrm{CDCl}_{3}\right) 61.4\left(\mathrm{OCH}_{3}\right)$, $61.1\left(\mathrm{OCH}_{3}\right), 56.5\left(\mathrm{OCH}_{3}\right), 109.2(\mathrm{C}-6), 115.2(\mathrm{C}-1), 141.0(\mathrm{C}-3), 146.3(\mathrm{C}-4), 150.3(\mathrm{C}-2)$, 151.8 (C-5), $194.9(\mathrm{CHO}) ; \mathrm{IR}\left(\mathrm{KBr}, \mathrm{cm}^{-1}\right) 1641(\mathrm{C}=\mathrm{O}), 3240$ (br, OH); MS (EI): $m / z 213$ [M+H $\left.{ }^{+}\right]$.

2-Hydroxy-4,5-dimethoxybenzaldehyde (24, $\left.\mathrm{C}_{9} \mathrm{H}_{10} \mathrm{O}_{3}\right)$. Yellow solid. Yield: 85\%. mp $\left({ }^{\circ} \mathrm{C}\right)$ : 105.5-112.8. ${ }^{1} \mathrm{H}-\mathrm{NMR}\left(500 \mathrm{MHz}, \mathrm{CDCl}_{3}\right): 3.88\left(\mathrm{~s}, 3 \mathrm{H}, \mathrm{OCH}_{3}\right), 3.94\left(\mathrm{~s}, 3 \mathrm{H}, \mathrm{OCH}_{3}\right), 6.48(\mathrm{~s}, 1 \mathrm{H}, \mathrm{Ar}), 6.91(\mathrm{~s}, 1 \mathrm{H}$, $\mathrm{Ar}), 9.71(\mathrm{~s}, 1 \mathrm{H}, \mathrm{OH}), 11.40(\mathrm{~s}, 1 \mathrm{H}, \mathrm{CHO}) ;{ }^{13} \mathrm{C}-\mathrm{NMR}\left(75 \mathrm{MHz}, \mathrm{CDCl}_{3}\right) 56.4\left(\mathrm{OCH}_{3}\right), 56.4\left(\mathrm{OCH}_{3}\right)$, 100.1 (C-3), 112.9 (C-1), 113.2 (C-6), 143.0 (C-5), 157.2 (C-2), 159.4 (C-4), 194.0 (CHO); IR $\left(\mathrm{KBr}, \mathrm{cm}^{-1}\right) 1624(\mathrm{C}=\mathrm{O}), 3446(\mathrm{br}, \mathrm{OH}) ; \mathrm{MS}(\mathrm{EI}): \mathrm{m} / z 183\left[\mathrm{M}+\mathrm{H}^{+}\right]$.

\section{Conclusions}

A novel method for the total synthesis of six coumarins and five important intermediates has been developed. In most cases, the overall yields of these products are higher than those previously reported. Furthermore, this is the first reported total synthesis of 7-hydroxy-6-ethoxycoumarin (3) and 6,7,8-trimethoxycoumarin (4). All of these coumarins and advanced intermediates will be evaluated for their activity, and the results will be reported in due course.

\section{Acknowledgments}

This work was financially supported by Fundamental Research Funds for the Central Universities (DL12EA01) and the Heilongjiang Postdoctoral Scientific Research Fund (LBH-Q11182).

\section{Conflicts of Interest}

The authors declare no conflict of interest. 


\section{References}

1. Cubukcu, B.; Bray, D.H.; Warhurst, D.C.; Mericli, A.H.; Ozhatay, N.; Sariyar, G. In vitro antimarlarial activity of crude extracts and compounds from Artemisia abrotanum L. Phytother. Res. 1990, 4, 203-204.

2. Huang, H.-Y.; Ko, H.-H.; Jin, Y.-J.; Yang, S.-Z.; Shih, Y.-A.; Chen, I.-S. Dihydrochalcone glucosides and antioxidant activity from the roots of Anneslea fragrans var. lanceolata. Phytochemistry 2012, 78, 120-125.

3. Shafiullah, K.; Naheed, R.; Nighat, A.; Abdul, M.; Aziz-ur-Rehmana; Lubna, I.; Mehreen, L. Antixoidant constituent from Cotoneaster racemiflora. J. Asian Nat. Prod. Res. 2009, 11, 44-48.

4. Shaw, C.-Y.; Chen, C.-H.; Hsu, C.-C.; Chen, C.-C.; Tsai, Y.-C. Antioxidant properties of scopoletin isolated from Sinomonium acutum. Phytother. Res. 2003, 17, 823-825.

5. Thuong, P.T.; Hung, T.M.; Ngoc, T.M.; Ha, D.T.; Min, B.S.; Kwack, S.J.; Kang, T.S.; Choi, J.S.; Bae, K. Antioxidant activity of coumarins from Korean medicinal plants and their structure-activity relationships. Phytother. Res. 2010, 24, 101-106.

6. Deianaa, M.; Rosaa, A.; Casua, V.; Cottigliab, F.; Bonsignoreb, L.; Dessìa, M.A. Chemical composition and antioxidant activity of extracts from Daphne gnidium L. J. Am. Oil Chem. Soc. 2003, 80, 65-70.

7. Smyth, T.; Ramachandran, V.N.; Smyth, W.F. A study of the antimicrobial activity of selected naturally occurring and synthetic coumarins. Int. J. Antimicrob. Ag. 2009, 33, 421-426.

8. Niu, X.; Xing, W.; Li, W.; Fan, T.; Hu, H.; Li, Y. Isofraxidin exhibited anti-inflammatory effects in vivo and inhibited TNF- $\alpha$ production in LPS-induced mouse peritoneal macrophages in vitro via the MAPK pathway. Int. Immunopharmacol. 2012, 14, 164-171.

9. Jiménez-Orozco, F.A.; Rosales, A.A.R.; Vega-López, A.; Domínguez-López, M.L.; García-Mondragón, M.J.; Maldonado-Espinoza, A.; Lemini, C.; Mendoza-Patiño, N.; Mandoki, J.J. Differential effects of esculetin and daphnetin on in vitro cell proliferation and in vivo estrogenicity. Eur. J. Pharmacol. 2011, 668, 35-41.

10. Liu, H.; Li, Z.; Yu, L.; Zhang, Y. Antitumor activity and mechanisms of scoparone. J. Zhong Guo Yao Li Tong Xun 2005, 22, 40-41.

11. Liu, W.; Hua, J.; Zhou, J.; Zhang, H.; Zhu, H.; Cheng, Y.; Gust, R. Synthesis and in vitro antitumor activity of novel scopoletin derivatives. Bioorg. Med. Chem. Lett. 2012, 22, 5008-5012.

12. Rouesac, F.; Leclerc, A. An efficient synthesis of isofraxidin. Synth. Commun. 1993, 23, 1147-1153.

13. Chen, W. Total synthesis of isofraxidin. Chin. J. Med. Chem. 1996, 6, 269-271.

14. Chen, W.A. Convenient total synthesis of isofraxidin, a natural coumarin. Indian J. Chem. Sect. B Org. Chem. Incl. Med. Chem. 1996, 35, 1085-1087.

15. Demyttenaere, J.; Syngel, K.V.; Markusse, A.P.; Vervisch, S.; Debenedetti, S.; de Kimpe, N. Synthesis of 6-methoxy-4H-1-benzopyran-7-ol, a character donating component of the fragrance of Wisteria sinensis. Tetrahedron 2002, 58, 2163-2166.

16. Crosby, D.G. Improved synthesis of scopoletin. J. Org. Chem. 1961, 26, 1215-1217.

17. Hauer, H.; Ritter, T.; Grotemeier, G. An improved and large scale synthesis of the natural coumarin scopoletin. Arch. Pharm. 1995, 328, 737-738. 
18. Hao, S.; Zong, G.; Wei, Y.; Meng, Z. Synthesis and pesticidal activity of 6,7-dimethoxy coumarin. J. Qingdao Agric. Univ. 2007, 24, 241-244.

19. Zhang, S.; Ma, J.; Chen, S.; Li, H.; Xin, J. Improved synthesis techniques of 6,7-dimethoxy coumarin. J. Hebei Univ. Sci. Technol. 2007, 28, 24-28.

20. Tanaka, H.; Kato, I.; Ito, K. Total synthesis of daphnetin, a coumarin lignold. Chem. Pharm. Bull. 1986, 34, 628-632.

21. Ahmed, B.; Khan, S.A.; Alam, T. Synthesis and antihepatotoxic activity of some heterocyclic compounds containing the 1,4-dioxane ring system. Pharmazie 2003, 58, 173-176.

22. Curini, M.; Epifano, F.; Maltese, F.; Marcotullio, M.C.; Gonzales, S.P.; Rodriguez, C. Synthesis of collinin an antiviral coumarin. Aust. J. Chem. 2003, 56, 59-60.

23. Aslam, K.; Khosa, M.K.; Jahan, N.; Nosheen, S. Synthesis and application of coumarin. Pak. J. Pharm. 2010, 23, 449-454.

24. Mahesh, K.P.; Swapnil, S.M.; Manikrao, M.S. Coumarin synthesises via Pechmann condensation in Lewis acidic chloroaluminate ionic liquid. Tetrahedron Lett. 2001, 42, 9285-9287.

25. Shaabani, A.; Ghadari, R.; Rahmati, A.; Rezayan, A.H. Coumarin synthesis via Knoevenagel Condensation Reation in 1,1,3,3-N,N,N'N'-tetramethylguanidinium trifluoroacetate ionic liquid. J. Iran Chem. Soc. 2009, 6, 710-714.

26. Harayama, T. Synthtic studies on aromatic heterocyclic compounds. Pharm. Soc. Japan 2006, $126,543-564$.

27. Kraus, G.A.; Cui, W.; Seo, Y.H. The reaction of Aryl triflates and Aryl pivalates with electrophiles. The triflate as a meta-directing group. Tetrahedron Lett. 2002, 43, 7077-7078.

28. Castanet, A.-S.; Colobert, F.; Broutin, P.-E. Mild and regioselective iodination of electron-rich aromatics with $\mathrm{N}$-iodosuccinimide and catalytic trifluoroacetic acid. Tetrahedron Lett. 2002, 43, 5047-5048.

29. Olah, G.A.; Wang, Q.; Sandford, G.; Surya, P.G.K. Iodination of deactivated aromatics with $N$-iodosuccinimide in trifluoromethanesulfonic acid $\left(\mathrm{NIS}-\mathrm{CF}_{3} \mathrm{SO}_{3} \mathrm{H}\right)$ via in situ generated superelectrophilic iodine (I) trifluoromethanesulfonate. J. Org. Chem. 1993, 58, 3194-3195.

30. Yang, D.; Fu, H. A simple and practical copper-catalyzed approach to substituted phenols from Aryl halides by using water as the solvent. Chem. Eur. J. 2010, 16, 2366-2370.

31. Qi, S.; Wu, D.; Luo, X. Coumarins and ellagic acids from sapium chihsinianum S. Lee. Nat. Prod. Res. Dev. 2004, 16, 297-299.

Sample Availability: Samples of the compounds 1-6 are available from the authors.

(C) 2013 by the authors; licensee MDPI, Basel, Switzerland. This article is an open access article distributed under the terms and conditions of the Creative Commons Attribution license (http://creativecommons.org/licenses/by/3.0/). 